\title{
Editorial: models of invertebrate neurons in culture
}

\author{
Amir Ayali \\ Received: 31 March 2012/Accepted: 3 April 2012/Published online: 15 April 2012 \\ (C) Springer Science+Business Media B.V. 2012
}

In an attempt to better model the human nervous system, experimental preparations in current neuroscience have become increasingly complex: Advances in recording and imaging techniques as well as in analysis methods, enable the study of the mammalian nervous systems at a greater resolution than ever previously possible (e.g. Baker 2010; Stevenson and Kording 2011), and allows investigation of intricate phenomena in higher vertebrate models.

At the same time, however, with the renaissance of the notion of conserved principles in neurobiology (e.g. Ache and Young 2005; Marder and Bucher 2007; Hobert et al. 2010; see Greenspan 2005 and references within), there has been a revival of interest in invertebrate models in neuroscience. There are striking similarities between species in regard to basic neural features, from the nature of relevant proteins, to cellular processes, to the organization of neuronal networks and pathways, through neural computation and dynamic processes leading to behavior (e.g. Godenschwege et al. 2006; Yanay et al. 2008; Humeau et al. 2011; and see Lichtneckert and Reichert 2005; Arendt et al. 2008; Brand and Livesey 2011; for reviews). These common features span a phylogenetically broad array of animals, vertebrate and invertebrate alike, implying both common ancestors and optimal-homologue solutions to similar problems (Clarac and Pearlstein 2007; Humeau et al. 2011; Chittka and Skorupski 2011 and references within).

Similarly to the above, a second duality characterizes current research; Together with important breakthroughs in the ability to conduct in vivo studies (e.g. Tsytsarev et al. 2006; Cardin et al. 2010), there is continued interest in the

\footnotetext{
A. Ayali $(\square)$

Department of Zoology, Tel-Aviv University,

69978 Tel-Aviv, Israel

e-mail: ayali@tauex.tau.ac.il
}

unique advantages offered by in vitro models. The fundamental questions of how a collection of single entities (i.e. neurons and glia), organize to form a complex functional unit-the neural network, and how these building blocks connect and interact to form further elaborate neuronal structures are extremely tractable in two-dimensional in vitro preparations of primary neuronal cultures (e.g. Bulloch and Syed 1992; Jimbo et al. 2000; Shefi et al. 2002; Darya et al. 2009; and see Beadle 2006 for review of insect neural cultures). The in vitro system is simple (relative to any in vivo network), and allows control over as many of its variables as possible. Most importantly, two-dimensional cultures enable a close look at the dynamics of neural growth and network organization (Fig. 1) by offering easy access for non-invasive optical observations. These processes can also be manipulated by various methods, such as genetic treatments (Bai et al. 2009; Tessier and Broadie 2011), pharmacological interventions (Perk and Mercer 2006; Heck et al. 2009), and the use of patterned growth surfaces (Liu et al. 2000; Anava et al. 2009), all of which can affect the neural network development and activity. The experimental results, or the rules discovered in these model systems, can then be translated to theoretical predictions and simple model assumptions, in order for them to be tested and applied to more complex systems.

This special issue of the Journal of Molecular Histology combines and builds upon the different trends mentioned above by focusing on in vitro preparations of invertebrate neurons.

Neurons of various invertebrates in primary culture have served as the preparation of choice in numerous studies, focusing on nervous system development, form-function interactions, neural pharmacology and many more (e.g. Acklin and Nichlls 1990; Hayashi and Hildebrand 1990; Howes et al. 1991; Kirchhof and Bicker 1992; Lapied et al. 

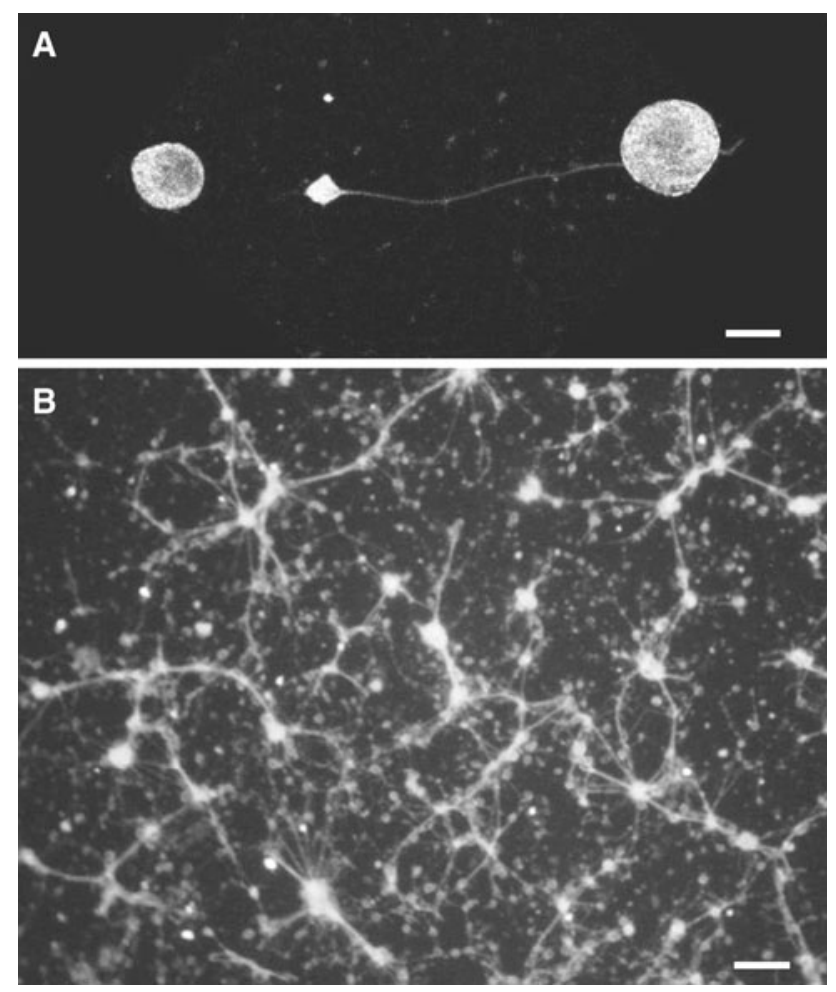

Fig. 1 a Locust neurons in culture as a model for neurite regeneration, growth cone navigation, and cell-cell interactions. Cell dissociated from the frontal ganglion, range in diameter from 20 to $50 \mu \mathrm{m}$. Scale bar $20 \mu \mathrm{m}$ (Anava and Ayali, unpublished). b Drosophila neurons in culture demonstrate intricate network topology, cell migration, and cluster formation. Scale bar $50 \mu \mathrm{m}$ (Saad and Ayali, unpublished)

1993; Whitington 1993; Kloppenburg and Horner 1998; Fromherz 1999; and see current issue). Accordingly, these experimental preparations have made a major contribution to our current knowledge in these diverse fields. In vitro twodimensional cultures of invertebrate neurons offer an attractive preparation for study, because of the large size of the cells and the facility with which they can be cultured under various conditions (e.g. extremely low densities). Furthermore, many invertebrate neurons are identifiable neurons (Leonard 2000), which allows identifying neurons in situ and the comparative characterization of neuronal structure and network circuitry in vitro (in culture) and in vivo.

This special issue provides a venue through which a panorama of current and cutting-edge neurobiological research is presented. Prospective authors with diverse research approaches were invited to contribute and we are delighted by the enthusiastic response. All submissions, including broad reviews, targeted reviews, and primary experimental papers were peer-reviewed. The result is a superb collection of articles, representing a wide variety of research areas with one thing in common, the important contribution of invertebrate neurons in culture as models in modern neuroscience.
Following is a short review of the contributions included in this special issue:

Schmold and Syed offer an in-depth review of the contribution of in vitro preparations from non-classical models (i.e. other than mouse, worm, or fly). Focusing largely on molluscs and leeches, the authors discuss learning, memory, and the concept of "identified" neurons, overall suggesting the advantages of using cultured invertebrate neurons as a model to study the fundamentals of nervous system function in higher animals.

Keeping to the mollusk neurons theme, Saada-Madar et al. present unusual features of the well-known pattern initiator neurons B31/B32 of Aplysia, when these neurons are cultured in isolation. The in vitro preparation assists in unveiling cellular properties and modulatory mechanisms, which are likely to contribute to the control of behavior in the animal.

An additional mollusk culture preparation, cultivated larval cells of the mussel Mytilus trossulus, is presented by Odintsova and Maiorova. These authors study cell adhesion and neuronal and muscle differentiation, and specifically the role of integrin-dependent mechanisms in these processes.

Insect neurons in culture have been amply studied and are accordingly well represented here. Ellen and Mercer review results obtained from studies of cultured insect antennal lobe neurons, suggesting an important modulatory role of the biogenic amines, dopamine and serotonin, in the regulation of neuronal development and promotion of cellular and behavioral plasticity.

Locusts are a leading preparation in the study of many neurophysiology and neuro-endocrinology-related questions. Locust neurons in culture have been used to investigate various questions of nervous system development, from cellular, and even molecular, to network-level aspects. The paper by Weigel et al. offers an important further step, presenting the locust primary neuronal culture as a model for the study of synaptic transmission.

Saad and Ayali report their results promoting a Drosophila primary neural culture preparation as a promising platform for studying a variety of processes related to nervous system development, activity, and pathology. The very well characterized in vitro network development, together with the well-known advantages offered by fly genetics, has great potential for future work.

Last but not least, further extending the presented repertoire of invertebrate models, Baranes et al. present a culture preparation of leech neurons plated on substrates photolithography-fabricated with repeatable nano-scale line-patterned ridges. Using high resolution electron microscopy, they demonstrate interactions of the neuronal processes with the nano-cues that affect neuronal morphology and neuronal branching topology. 
To conclude, the varied collection of articles in this special issue offer readers the opportunity to be introduced to a diversity of research interests, methodologies, and approaches. The common theme or thread connecting these different contributions and research groups is a strong belief in the important contribution of invertebrate neurons in culture preparations to contemporary neuroscience.

I appreciate the timely submission of interesting manuscripts by the contributors. I am grateful to Martijn Roelandse and Srilakshmi Patrudu for invaluable help in assembling this special Issue.

\section{References}

Ache BW, Young JM (2005) Olfaction: diverse species, conserved principles. Neuron 48:417-430. doi:10.1016/j.neuron.2005.10.022

Acklin SE, Nichlls JG (1990) Intrinsic and extrinsic factors influencing properties and growth-patterns of identified leech neurons in culture. J Neurosci 10:1082-1090

Anava S, Greenbaum A, Ben Jacob E et al (2009) The regulative role of neurite mechanical tension in network development. Biophys J 96:1661-1670. doi:10.1016/j.bpj.2008.10.058

Arendt D, Denes AS, Jékely G, Tessmar-Raible K (2008) The evolution of nervous system centralization. Philos Trans R Soc Lond Ser B Biol Sci 363:1523-1528. doi:10.1098/rstb.2007. 2242

Bai J, Sepp KJ, Perrimon N (2009) Culture of Drosophila primary cells dissociated from gastrula embryos and their use in RNAi screening. Nat Protoc 4:1502-1512. doi:10.1038/nprot.2009.147

Baker M (2010) From promising to practical: tools to study networks of neurons. Nat Methods 7:877-883. doi:10.1038/nmeth1110-877

Beadle DJ (2006) Insect neuronal cultures: an experimental vehicle for studies of physiology, pharmacology and cell interactions. Invert Neurosci 6:95-103

Brand AH, Livesey FJ (2011) Neural stem cell biology in vertebrates and invertebrates: more alike than different? Neuron 70:719729. doi:10.1016/j.neuron.2011.05.016

Bulloch AGM, Syed NI (1992) Reconstruction of neuronal networks in culture. Trends Neurosci 15:422-427

Cardin JA, Carlén M, Meletis K et al (2010) Targeted optogenetic stimulation and recording of neurons in vivo using cell-typespecific expression of Channelrhodopsin-2. Nature Protoc 5:247-254. doi:10.1038/nprot.2009.228

Chittka L, Skorupski P (2011) Information processing in miniature brains. Proc Biol Sci R Soc 278:885-888. doi:10.1098/rspb. 2010.2699

Clarac F, Pearlstein E (2007) Invertebrate preparations and their contribution to neurobiology in the second half of the 20th century. Brain Res Rev 54:113-161. doi:10.1016/j.brainresrev. 2006.12.007

Darya K, Ganguly A, Lee D (2009) Quantitative analysis of synaptic boutons in Drosophila primary neuronal cultures. Brain Res 1280:1-12. doi:10.1016/j.brainres.2009.05.026

Fromherz P (1999) Extracellular recording with transistors and the distribution of ionic conductances in a cell membrane. Eur Biophys J 28:254-258

Godenschwege TA, Kristiansen LV, Uthaman SB et al (2006) A conserved role for Drosophila Neuroglian and human L1-CAM in central-synapse formation. Current Biol CB 16:12-23. doi: 10.1016/j.cub.2005.11.062
Greenspan RJ (2005) No critter left behind: an invertebrate renaissance. Curr Biol CB 15:R671-R672. doi:10.1016/j.cub.2005.08.018

Hayashi JH, Hildebrand JG (1990) Insect olfactory neurons invitromorphological and physiological characterization of cells from the developing antennal lobes of Manduca sexta. J Neurosci 10:848-859

Heck C, Kunst M, Härtel K et al (2009) In vivo labeling and in vitro characterisation of central complex neurons involved in the control of sound production. J Neurosci Methods 183:202-212. doi:10.1016/j.jneumeth.2009.06.032

Hobert O, Carrera I, Stefanakis N (2010) The molecular and gene regulatory signature of a neuron. Trends Neurosci 33:435-445. doi:10.1016/j.tins.2010.05.006

Howes EA, Cheek TR, Smith PJS (1991) Long-term growth in vitro of isolated, fully differentiated neurones from the central nervous system of an adult insect. J Exp Biol 156:591-605

Humeau Y, Candiani S, Ghirardi M et al (2011) Functional roles of synapsin: lessons from invertebrates. Semin Cell Dev Biol 22:425-433. doi:10.1016/j.semcdb.2011.07.018

Jimbo Y, Kawana A, Parodi P, Torre V (2000) The dynamics of a neuronal culture of dissociated cortical neurons of neonatal rats. Biol Cybern 83(1):1-20

Kirchhof B, Bicker G (1992) Growth properties of larval and adult locust neurons in primary cell culture. J Comp Neurol 323: $411-422$

Kloppenburg P, Horner M (1998) Voltage-activated currents in identified giant interneurons isolated from adult crickets Gryllus bimaculatus. J Exp Biol 201:2529-2541

Lapied B, Tribut F, Sinakevitch I, Hue B, Beadle DJ (1993) Neurite regeneration of long-term cultured adult insect neurosecretorycells identified as dum neurons. Tissue Cell 25:893-906

Leonard JL (2000) Identifiable neurons in invertebrates: from invariant cells to dynamic systems-Preface. Brain Behav Evol 55:233-235. doi:10.1159/000006656

Lichtneckert R, Reichert H (2005) Insights into the urbilaterian brain: conserved genetic patterning mechanisms in insect and vertebrate brain development. Heredity 94:465-477. doi:10.1038/sj. hdy.6800664

Liu QY, Coulombe M, Dumm J et al (2000) Synaptic connectivity in hippocampal neuronal networks cultured on micropatterned surfaces. Brain Res Dev Brain Res 120:223-231

Marder E, Bucher D (2007) Understanding circuit dynamics using the stomatogastric ganglion of lobsters and crabs. Annu Rev Physiol 69:291-316

Perk CG, Mercer AR (2006) Dopamine modulation of honey bee (Apis mellifera) antennal-lobe neurons. J Neurophysiol 95: 1147-1157. doi:10.1152/jn.01220.2004

Shefi O, Ben-Jacob E, Ayali A (2002) Growth morphology of twodimensional insect neural networks. Neurocomputing 44-46: 635-643

Stevenson IH, Kording KP (2011) How advances in neural recording affect data analysis. Nat Neurosci 14:139-142. doi:10.1038/nn. 2731

Tessier CR, Broadie K (2011) The fragile X mental retardation protein developmentally regulates the strength and fidelity of calcium signaling in Drosophila mushroom body neurons. Neurobiol Dis 41:147-159. doi:10.1016/j.nbd.2010.09.002

Tsytsarev V, Taketani M, Schottler F et al (2006) A new planar multielectrode array: recording from a rat auditory cortex. J Neural Eng 3:293-298. doi:10.1088/1741-2560/3/4/006

Whitington PM (1993) Axon guidance factors in invertebrate development. Pharmacol Ther 58:263-299

Yanay C, Morpurgo N, Linial M (2008) Evolution of insect proteomes: insights into synapse organization and synaptic vesicle life cycle. Genome Biol 9:R27. doi:10.1186/gb-2008-9-2-r27 